\title{
Protective Effects of Akebia Saponin D Against Rotenone-Induced Hepatic Mitochondria Dysfunction
}

\author{
Li-li Gong ${ }^{1}$, Zi-hui Wang ${ }^{1}$, Guang-run $\mathrm{Li}^{1}$, and Li-hong Liu ${ }^{1, *}$ \\ ${ }^{1}$ Beijing Chao-Yang Hospital, Capital Medical University, Beijing 100020, China
}

Received May 30, 2014; Accepted September 3, 2014

\begin{abstract}
Akebia saponin D (ASD) is a typical bioactive triterpenoid saponin obtained from the rhizome of Dipsacus asper Wall. Previous studies have found that ASD has a hepatoprotective effect in a mouse model. The purpose of this paper was to explore the molecular mechanism of the hepatoprotective effects of ASD on BRL cells and isolated rat liver mitochondria. We investigated the effects of ASD on rotenone-induced toxicity in BRL cells. The results showed that ASD inhibited the accumulation of reactive oxidant species, ATP deficiency, and mitochondrial membrane potential dissipation; ameliorates mitochondrial respiratory dysfunction, and improved the activity of complex I in a concentration-dependent manner, indicating that ASD likely improved mitochondrial function. ASD suppressed rotenone-induced BRL cell apoptosis and increased Bcl-2/Bax ratio. These results suggest that ASD may exert hepatoprotective effects against rotenone-induced toxicity through mitochondria. This study supports our previous research that ASD possesses hepatoprotective activity in vivo and it is worthy of further study.
\end{abstract}

Keywords: Akebia saponin D, mitochondria, apoptosis, rotenone

\section{Introduction}

Death of hepatocytes is a characteristic feature of liver diseases as diverse as cholestasis, viral hepatitis, ischemia/reperfusion, liver preservation for transplantation, and drug/toxicant-induced injury (1). Hepatic cell death is the leading cause of fatality in patients with liver diseases. Liver is a major metabolic site for xenobiotics and is known to be the primary target organ targeted by variety of chemicals/drugs (2). Rotenone, a natural pesticide and insecticide, is a specific inhibitor of complex I of the mitochondrial respiratory chain. Inhibition of the mitochondrial respiratory chain by rotenone had been found to induce cell death in a variety of cells (3). The mechanisms of rotenone-induced apoptosis involved several aspects such as inhibition of the electron transport system through respiratory chain complex I (4), decreasing cellular ATP level (5), increasing mitochondrial ROS production (3), and decreasing mitochondrial membrane potential (6). Rotenone has been widely

*Corresponding author. hongllh@126.com

Published online in J-STAGE on October 15, 2014

doi: 10.1254/jphs.14135FP used to induce apoptosis in liver cells. BRL cells, a cell line established from Buffalo rat liver, rich in mitochondria, have been widely used for liver biological and chemical studies. Rotenone induced BRL cell models have been used to screen compounds that have potential hepatoprotection.

Dipsacus asper Wall (DAW), which belongs to Dipsacaceae, is a perennial herb growing in moist fields and mountains. The roots of DAW have been used for hundreds of years as an anti-osteoporosis, tonic, and antiaging agent in China for the therapy of low back pain, traumatic hematoma, threaten abortion, and bone fractures (7). As a typical compound of DAW, the protective effect of Akebia saponin D (ASD) (Fig. 1) has been described in some disease models such as cancer (8), Alzheimer's disease $(7,9)$, cardiovascular disease $(2,10,11)$, and bone fractures $(12,13)$. Our previous studies have shown that ASD has a hepatoprotective effect in a mouse model (14). However, the molecular mechanism of hepatoprotective effects of ASD has not been clearly established and it is uncertain whether or not the effect of ASD is mediated by the action on mitochondria. In the present study, we studied the protective action of ASD against mitochondria-mediated 


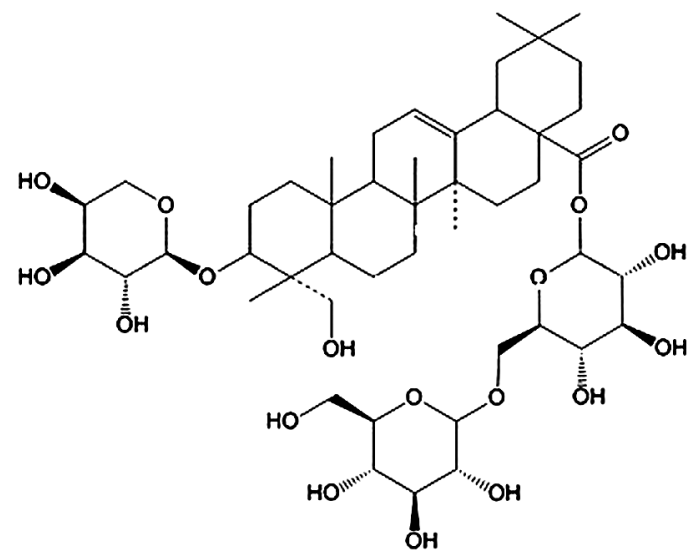

Fig. 1. The chemical structure of Akebia saponin D.

apoptosis induced by rotenone in BRL cells. The direct effects of ASD on rotenone-treated and normal rat liver mitochondrial were also studied.

\section{Materials and Methods}

\section{Drugs and reagents}

ASD was extracted by Beijing Chao-Yang Hospital affiliated with Beijing Capital Medical University and its structure was confirmed on the basis of physicalchemical properties and spectral evidence (15). The purity of ASD was more than $98 \%$ as tested by the HPLC method. Rotenone was purchased from SigmaAldrich (St. Louis, MO, USA).

\section{Studies in BRL cells}

Cell culture and treatment: BRL cells were purchased from the Cell Center of Shanghai Institutes for Biological Sciences (Shanghai, China). Cells were grown in Dulbecco's Modified Eagle Medium (DMEM) supplemented with $10 \%$ FBS and kept at $37^{\circ} \mathrm{C}$ in a $5 \% \mathrm{CO}_{2}$ incubator. BRL cells were pretreated with various concentrations of ASD for $1 \mathrm{~h}$, followed by incubating with $2 \mu \mathrm{M}$ rotenone or vehicle control for $24 \mathrm{~h}$.

Cell viability assay: Cell viability was assessed by the MTT assay. After $24 \mathrm{~h}$ of culture, $20 \mu \mathrm{L}$ of MTT solution $\left(0.5 \mathrm{mg} \cdot \mathrm{mL}^{-1}\right)$ was added to each well and incubated for $4 \mathrm{~h}$. The supernatant was removed, and the formazan crystals were dissolved in $200 \mu \mathrm{L}$ of dimethyl-sulfoxide. The absorbance at $490 \mathrm{~nm}$ was determined using a microplate reader (SpectraMax M5e; Molecular Devices, Sunnyvale, CA, USA).

Lactate dehydrogenase (LDH) release, an index of the cell membrane integrity, was measured using the Cytotoxicity Detection Kit Plus (Roche Diagnostics, Mannheim, Germany) according to the manufacturer's instruction. Briefly, after $24 \mathrm{~h}$ of cultivation, $100 \mu \mathrm{L}$ reaction mixture was added to each well and incubated for $30 \mathrm{~min}$ at $22^{\circ} \mathrm{C}$ and then $50 \mu \mathrm{L}$ Stop Solution was added. The absorbance at $490 \mathrm{~nm}$ was measured.

Measurement of ATP level: ATP level was measured with the CellTiter-Glo luminescent cell viability kit from Promega Corporation (Madison, WI, USA) following the manufacturer's instructions. Briefly, after $24 \mathrm{~h}$ of cultivation, the plate was equilibrated to room temperature for $30 \mathrm{~min}$, and then $100 \mu \mathrm{L}$ reagent was added into each well. The contents were mixed for 2 min to induce cell lysis. After 10-min incubation at room temperature, the luminescence was read on a Microplate Reader (SpectraMax M5e; Molecular Devices).

Intracellular ROS measurement: Intracellular ROS were detected using the dye 2,7-dichlorodihydrofluorescein diacetate (DCFH2-DA, Molecular Probes) as a fluorescent probe. $\mathrm{DCFH}_{2}-\mathrm{DA}$ is a nonfluorescent compound, and it can be enzymatically converted to highly fluorescent compound, DCF, in the presence of ROS. BRL cells were treated with various concentrations of ASD and $2 \mu \mathrm{M}$ rotenone for $24 \mathrm{~h}$, washed with PBS, and incubated with $10 \mu \mathrm{M} \mathrm{DCFH}{ }_{2}-\mathrm{DA}$ at $37^{\circ} \mathrm{C}$ for 20 $\min$. DCF fluorescence was measured at $485 \mathrm{~nm}$ excitation and $520 \mathrm{~nm}$ emission (SpectraMax M5e; Molecular Devices).

Assessment of mitochondrial membrane potential: Mitochondrial membrane potential $(\Delta \psi \mathrm{m})$ was assessed by a fluorescent dye JC-1 (Beyotime Biotechnology, Jiangsu, China). Briefly, after $24 \mathrm{~h}$ of cultivation, cells were stained with $\mathrm{JC}-1\left(5 \mu \mathrm{g} \cdot \mathrm{mL}^{-1}\right)$ at $37^{\circ} \mathrm{C}$ for $20 \mathrm{~min}$. Then cells were rinsed twice with JC-1 staining buffer. Fluorescence was monitored in a spectrofluorometer using 490/535 $\mathrm{nm}$ for the monomeric form and 570/595 $\mathrm{nm}$ for the aggregate of JC-1. The fluorescence intensity ratio of aggregates to monomers was calculated as an indicator of mitochondrial membrane potential. The red/green fluorescence was also visualized with an Olympus fluorescence microscope.

Determination of nuclear damage: The nuclear morphological change was assessed using the Hoechst 33258. Nuclei were stained with Hoechst $33258\left(1 \mu \mathrm{g} \cdot \mathrm{mL}^{-1}\right)$ for $5 \mathrm{~min}$. Before imaging, cells were washed with PBS at least three times. Nuclei were visualized with an Olympus fluorescence microscope.

Detection of apoptosis by flow cytometry: Apoptosis was determined by an Annexin V-FITC Apoptosis Detection Kit followed by flow cytometric analysis. In brief, Cells were trypsinized and collected by centrifugation at $1000 \times g$ for $5 \mathrm{~min}$. After resuspension in annexin $\mathrm{V}-$ FITC binding buffer, cells were incubated with $1 \mu \mathrm{g} \cdot \mathrm{mL}^{-1}$ annexin V-FITC and $10 \mu \mathrm{g} \cdot \mathrm{mL}^{-1} \mathrm{PI}$ at room temperature in the dark for $15 \mathrm{~min}$. Samples were analyzed with a 
Kaluza flow cytometry. The samples were analyzed using an EPICS Profile II flow cytometry (Coulter, Hialeah, FL, USA).

Western blot analysis for bax and bcl-2: Cell lysates were subjected to SDS-PAGE and electrophoretically transferred to a polyvinylidene difluoride (PVDF) membrane. The membranes were then exposed to primary anti-bodies (Bax, Bcl-2, and $\beta$-actin; Cell Signaling Technology, Danvers, MA, USA) overnight at $4^{\circ} \mathrm{C}$. After incubation with the HRP-linked secondary antibody (Santa Cruz Biotechnology, Carlsbad, CA, USA) for $1 \mathrm{~h}$ at room temperature, immunoreactive proteins were visualized by enhanced chemiluminescence reagent (Molecular Imager ChemiDoc XRS+ System, Bio-Rad, Hercules, CA, USA).

\section{Studies on isolated rat liver mitochondria}

Animals: Six male Sprague-Dawley rats $(200 \pm 20$ g; Beijing Vital River Laboratory Animal Technology; Beijing, China; license: SCXK (JING) 2012-0001) were used in this study. All experiments were carried out in accordance with China Animal Welfare Legislation and were approved by Beijing Chao-Yang Hospital Committee on Ethics in the Care and Use of Laboratory Animals.

Preparation of rat liver mitochondria: The mitochondria were isolated as previously described with slight modifications(16). Briefly, rats were anesthetized and the liver was placed in beakers containing ice-cold isolation buffer $(250 \mathrm{mM}$ sucrose, $10 \mathrm{mM}$ Tris- $\mathrm{HCl} \mathrm{pH}$ 7.4, $1 \mathrm{mM}$ EDTA, and $0.25 \mathrm{mg} \cdot \mathrm{mL}^{-1}$ BSA). The liver tissue was repeatedly washed with the isolation buffer to remove adhering blood and $10 \%(\mathrm{w} / \mathrm{v})$ homogenate was prepared in a glass homogenizer. The nuclei and cell debris were sedimented by centrifugation at $600 \times g$ for $10 \mathrm{~min}$ and discarded. Mitochondria pellets were obtained after centrifugation at $12,000 \times \mathrm{g}$ for $10 \mathrm{~min}$ (Allegra X-22 Series Benchtop Centrifuge, Beckman Coulter, Ft. Detrick, MD, USA). The mitochondria pellet was suspended in the buffer at a concentration of $20 \mathrm{mg} \cdot \mathrm{mL}^{-1}$ and stored on ice for measurement. Mitochondrial protein quantification was determined by the Bradford method using BSA as the standard.

In the analysis of respiration, mitochondria $(0.5$ $\mathrm{mg} \cdot \mathrm{mL}^{-1}$ ) were incubated for $1 \mathrm{~min}$ in a Clark oxygen electrode. Accordingly, $2 \mu \mathrm{M}$ rotenone in the presence or absence of ASD was added before energization with respiration substrate and ADP. In other experiments, isolated mitochondria $\left(0.5 \mathrm{mg} \cdot \mathrm{mL}^{-1}\right)$ were pretreated with ASD for $30 \mathrm{~min}$ at $30^{\circ} \mathrm{C}$, followed by exposure to $2 \mu \mathrm{M}$ rotenone for another $30 \mathrm{~min}$.

Measurement of mitochondrial respiratory activity: Respiration measurements were carried out using a Clark oxygen electrode (Strathkelvin Instruments, Glasgow, UK). The reaction was carried out at $30^{\circ} \mathrm{C}$ in $1.0 \mathrm{~mL}$ respiration medium consisting of $225 \mathrm{mM}$ sucrose, $5 \mathrm{mM}$ potassium phosphate buffer $\mathrm{pH}$ 7.4, $10 \mathrm{mM}$ Tris- $\mathrm{HCl}$ buffer, $10 \mathrm{mM} \mathrm{KCl}, 0.2 \mathrm{mM}$ EDTA, and $100 \mathrm{mg} \cdot \mathrm{L}^{-1}$ BSA. Mitochondrial respiration was initiated by adding $250 \mu \mathrm{M}$ ADP with $10 \mathrm{mM} \mathrm{L}$-glutamate plus $5 \mathrm{mM} \mathrm{L}-$ malate or $10 \mathrm{mM}$ succinate as the NADH or FAD chain respiration substrate. State 3 respiration was defined as the oxygen consumption rate in the presence of added $\mathrm{ADP}$, and state 4 respiration was the rate of oxygen consumption following depletion of ADP. The respiration control ratio (RCR) was calculated as the ratio of state 3 respiration to state 4 respiration and used as a marker of mitochondrial respiration activity. The ADP/O ratio was calculated as the ratio of the added ADP concentration to the consumption of oxygen during state 3 respiration. The oxidative phosphorylation rate (OPR) was obtained from the product of state 3 respiration rate and $\mathrm{ADP} / \mathrm{O}$ ratio.

Measurement of ATP and ROS level: After incubation for $30 \mathrm{~min}$ with $2 \mu \mathrm{M}$ rotenone with or without ASD pretreatment, ATP content of mitochondria of different groups was assessed using a luciferase-coupled ATP quantification assay in accordance with the manufacturer's directions as previously described. ROS production in isolated rat liver mitochondria was monitored using the fluorescent probe $\mathrm{DCFH}_{2}$-DA as previously described.

Complex I, II, III, and IV activity measurement: The activity of mitochondrial Complex I was assessed by calculating the NADH oxidation rate. Mitochandria were added to potassium phosphate buffer $(50 \mathrm{mM})$ containing $5 \mathrm{mM}$ sodium azide and $50 \mathrm{mM}$ coenzyme $\mathrm{Q}_{0}$. After preincubation for $5 \mathrm{~min}$ at $37^{\circ} \mathrm{C}$, the reaction was initiated by the addition of NADH $(120 \mathrm{mM})$ and the rate of decrease in the absorbance was monitored at $340 \mathrm{~nm}$ for $2 \mathrm{~min}$. The NADH oxidation rate was calculated from the time-dependent decrease of the slope of absorbance using a combined NADH ubiquinone extinction coefficient of $6.22 \mathrm{mM}^{-1} \cdot \mathrm{cm}^{-1}$ (SpectraMax M5e, Molecular Devices).

The activity of complex II was determined by 2,6 dichlorophenolindophenol reduction. The assay mixture contained $50 \mu \mathrm{g} \cdot \mathrm{mL}^{-1}$ mitochondrial protein, $50 \mathrm{mM}$ $\mathrm{KH}_{2} \mathrm{PO}_{4}, 5 \mathrm{mM} \mathrm{NaN}_{3}, 0.5 \mathrm{mM}$ duroquinone, and $25 \mathrm{mM}$ dichlorophenolindophenol. The reaction was initiated by adding $20 \mathrm{mM}$ succinate, and the change in absorbance of dichlorophenolindophenol was measured at $605 \mathrm{~nm}\left(\varepsilon=21 \mathrm{mM}^{-1} \cdot \mathrm{cm}^{-1}\right)$.

Complex III activity was measured by following the reduction of cytochrome $\mathrm{c}$ by decylubiquinol. The assay mixture contained mitochondrial protein $\left(10 \mu \mathrm{g} \cdot \mathrm{mL}^{-1}\right)$, 
A

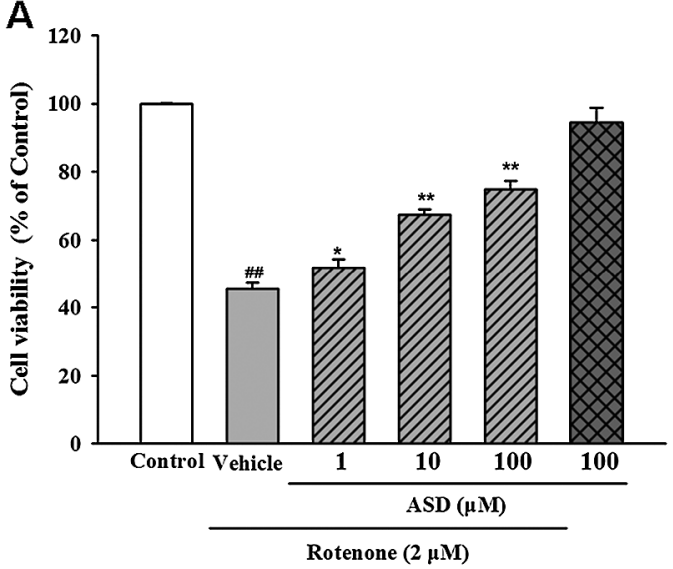

B

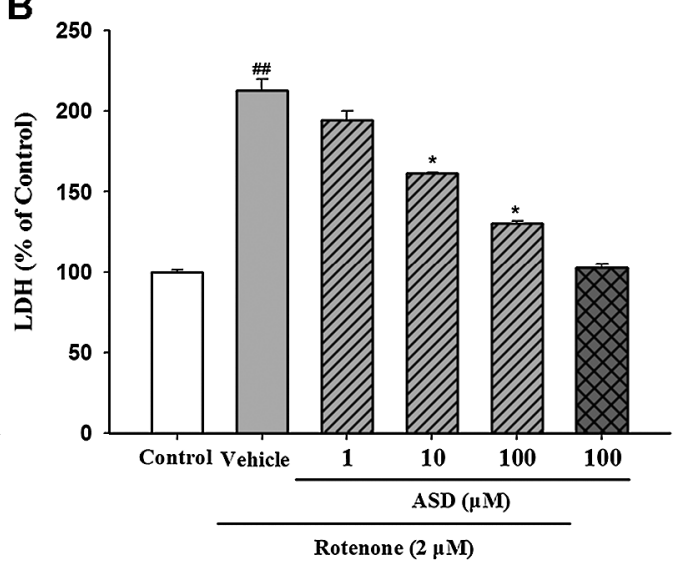

Fig. 2. Effects of Akebia saponin D (ASD) on rotenone-induced cytotoxicity in BRL cells. Cells were treated with $2 \mu \mathrm{M}$ rotenone in the presence or absence of ASD for $24 \mathrm{~h}$. A) Mitochondrial function was determined by MTT assay. B) LDH release was assessed in the medium of BRL cells. Data are expressed as means \pm S.E.M. $(n=6) .{ }^{\#} P<0.01$, compared with the control group; $* P<0.05, * * P<0.01$, compared with the rotenone group.

$50 \mathrm{mM} \mathrm{KH} \mathrm{KO}_{4}, 0.1 \mathrm{mM}$ EDTA, $0.1 \%$ BSA, $3 \mathrm{mM}$ $\mathrm{NaN}_{3}$, and $60 \mu \mathrm{M}$ oxidized cytochrome c. The reaction was started by adding $100 \mu \mathrm{M}$ decylubiquinol and the decrease in absorbance at $550 \mathrm{~nm}$ was followed. An extinction coefficient of $18.5 \mathrm{mM}^{-1} \cdot \mathrm{cm}^{-1}$ was used to calculate absolute changes.

The activity of complex IV was assessed by the decrease in the rate of absorbance of reduced cytochrome $\mathrm{c}$ at $550 \mathrm{~nm}\left(\varepsilon=18.5 \mathrm{mM}^{-1} \cdot \mathrm{cm}^{-1}\right)$. The assay mixture contained $50 \mathrm{mM} \mathrm{KH} \mathrm{PO}_{4}, 0.15 \mathrm{mg} \cdot \mathrm{mL}^{-1}$ asolectin, and $40 \mu \mathrm{M}$ reduced cytochrome $\mathrm{c}$. The reaction was initiated by adding $10 \mu \mathrm{g} \cdot \mathrm{mL}^{-1}$ of mitochondrial protein.

\section{Statistical analyses}

The results were expressed as means \pm S.E.M in each group. To analyze the differences between groups, initial analyses were conducted with one-way ANOVA tests followed by Dunnett's test. If the data did not fit the constraints of the parametric test, data were analyzed with ChiSquare Test, Kruskal-Wallis ANOVA, or the Mann-Whitney test. A value $P<0.05$ was considered statistically significant.

\section{Results}

\section{ASD attenuated rotenone induced cytotoxicity}

Cell viability was measured by MTT and LDH assay. After exposure to rotenone $(2 \mu \mathrm{M})$ for $24 \mathrm{~h}$, the cell viability decreased significantly. Pretreatment with ASD $(1,10,100 \mu \mathrm{M})$ attenuated rotenone induced cytotoxicity in a dose-dependent manner (Fig. 2).
ASD increased the cellular ATP levels and decreased oxidative stress

ATP level was quantified by using a luminescence assay. As shown in Fig. 3A, rotenone $(2 \mu \mathrm{M})$ resulted in a $61.5 \%$ decrease in ATP level. These changes were significantly suppressed by ASD $(1,10,100 \mu \mathrm{M})$ in a concentration-dependent manner.

Intracellular ROS were detected using a DCFH2-DA fluorescent probe. ROS production was increased in rotenone-treated cells compared to the control condition and this effect was abolished in the presence of ASD (Fig. 3B).

\section{ASD prevented rotenone induced membrane potential loss}

Mitochondrial membrane potential was analyzed by JC-1 fluorescence. As a result of rotenone treatment, cells displayed a loss of mitochondrial membrane potential $(67.30 \% \pm 0.70 \%$ ) (Fig. 4A). Rotenone significantly reduced the red fluorescence compared to controls (Fig. 4B). ASD prevents the breakdown of the mitochondrial membrane potential as indicated by preservation of the red JC-1 fluorescence.

\section{ASD suppressed rotenone induced apoptosis}

As shown in Fig. 5A, the majority of control group cells had uniformly stained nuclei after staining with Hoechst 33258. Exposure to $2 \mu \mathrm{M}$ rotenone for $24 \mathrm{~h}$ induced morphological changes typical of apoptosis (nuclei fragmentation with condensed chromatin and bright staining), as detected by fluorescent microscopy. The morphological changes indicating apoptosis in the nuclei of BRL cells were partly prevented by ASD. 
A

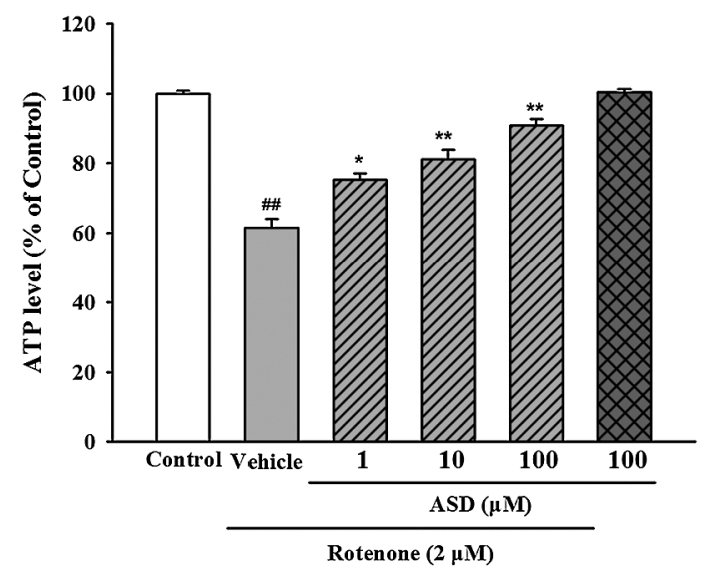

B

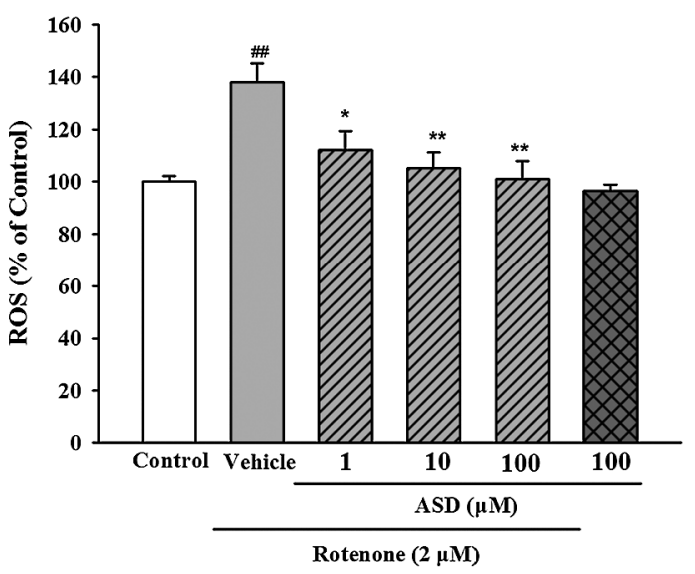

Fig. 3. Effects of Akebia saponin D (ASD) on rotenone induced ATP deficiency and reactive oxidant species production in BRL cells. A) Cells were treated with $2 \mu \mathrm{M}$ rotenone in the presence or absence of ASD for $24 \mathrm{~h}$. ATP level was assessed using a luciferase-coupled ATP quantification assay. Data are expressed as means \pm S.E.M. $(n=6)$. B) Cells were treated with $2 \mu$ M rotenone in the presence or absence of ASD for $24 \mathrm{~h}$. ROS production was evaluated by DCF fluorescence. Data are expressed as means \pm S.E.M. $(\mathrm{n}=6) .{ }^{\# \#} P<0.01$, compared with the control group; ${ }^{*} P<0.05,{ }^{* *} P<0.01$, compared with the rotenone group.

Annexin V-FITC / PI staining determined the percentage of early apoptotic cells (annexin V-positive/PInegative), late apoptotic cells (annexin V-positive/PIpositive), and necrotic cells (annexin V-negative/PIpositive). We found that cells treated with rotenone bound both annexin V and PI. Following the increasing of ASD, late apoptotic cells and necrotic cells were significantly decreased (Fig. 5B).

The Bcl-2 family members are regulated in response to cellular stress, including oxidative stress. Bcl-2 is an anti-apoptotic protein, whereas Bax is a proapoptotic protein. In this study, we investigated how the Bcl-2 and Bax proteins changed when treated with rotenone and whether ASD had any effect on their expressions. As shown in Fig. 6, A and B, the expressions of Bcl-2 proteins treated with ASD were significantly higher than that treated with rotenone. Bax protein expressions increased significantly in the rotenone group compared with that in the normal group. As shown in Fig. $6 \mathrm{C}$, the $\mathrm{Bcl}-2 / \mathrm{Bax}$ ratio in the rotenone group decreased to 0.59 -fold of the normal group, while ASD treatment effectively prevented this decrease to $0.81-, 1.26-$, and 2.05 -fold, respectively.

\section{Effects of ASD on liver mitochondrial respiration}

The results of mitochondrial respiration in the presence of glutamate plus malate or succinate were presented, respectively, in Table 1. The following parameters were determined: $\mathrm{RCR}, \mathrm{ADP} / \mathrm{O}$, state 3 respiration, state 4 respiration, and OPR. Activity of the NADH-linked respiratory chain was measured with malate/glutamate as substrate. The addition of $2 \mu \mathrm{M}$ rotenone caused a significant decline in state 3 respiration and state 4 respiration. Accordingly, the RCR value was approximately 1 , indicating that liver mitochondrial respiration was significantly inhibited. Treatment with ASD $(10 \mu \mathrm{M}, 100 \mu \mathrm{M})$ significantly restored the RCR, $\mathrm{ADP} / \mathrm{O}$, and OPR diminished by rotenone; however, $1 \mu \mathrm{M}$ ASD had no significant effect. When complex II substrate succinate was used, rotenone tended to increase the mitochondrial respiration compared with the control $(P>0.05)$. Treatment with ASD caused no significant change in mitochondrial respiration.

\section{Effects of ASD on liver mitochondrial energy metabolism and oxidative stress}

To observe the direct effect of ASD on mitochondrial energy metabolism and oxidative stress, ATP and ROS levels were measured in isolated rat liver mitochondria in further research. Addition of ASD exhibited significant protective effects on rotenone-induced ATP deficiency and ROS production, which were in accordance with the results obtained from the cellular study (Fig. 7: A, B).

\section{Effect of ASD on complex I, II, III, and IV activity}

Rotenone is a well-known specific complex I inhibitor of the mitochondrial electron transport chain. To determine the effect of ASD on rotenone toxicity, mitochondrial complex I activity was studied. Mitochondrial complex I activity was measured as NADH: ubiquinone oxidoreductase activity. As represented in Fig. 5A, 

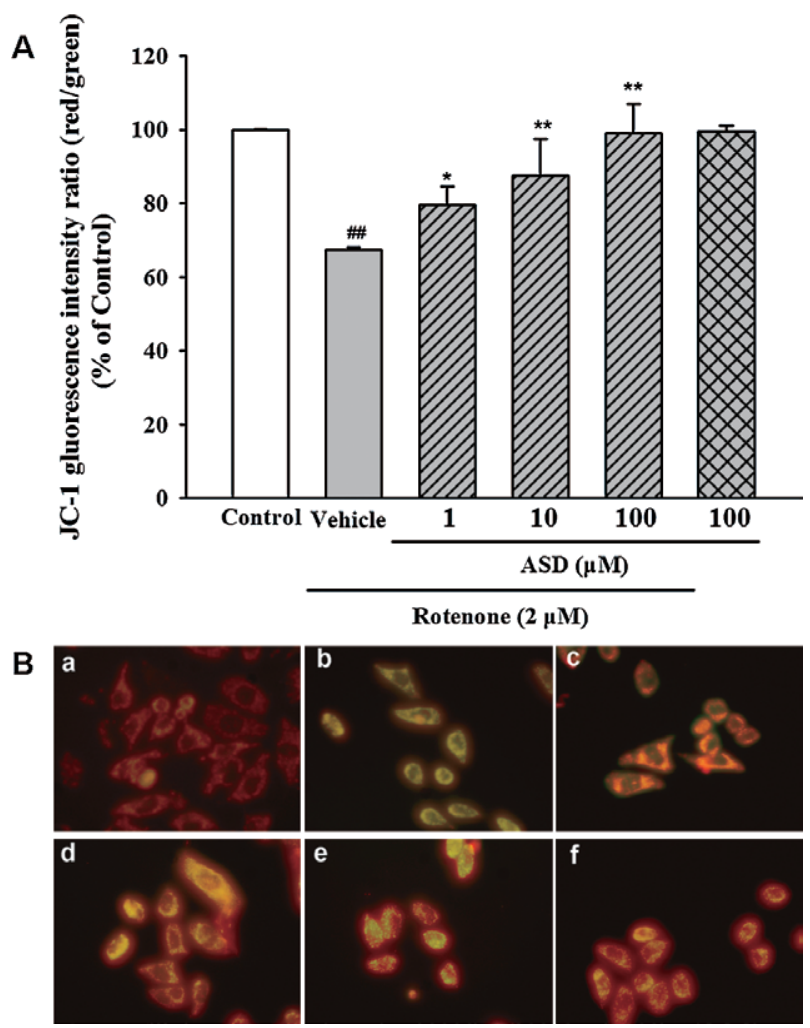

a major drop in Complex I activity was observed in the presence of rotenone. Co-incubation with ASD reversed the inhibitory effect of rotenone. The addition of rotenone did not cause a significant effect on the activities of complex II, III, and IV. Treatment with ASD also did not show a significant effect. However, Exposure of mitochondria to ASD alone caused some increase in the activities of complex II and complex III $(P>0.05)$ (Fig. 8).

Fig. 4. Effects of Akebia saponin D (ASD) on rotenone-induced mitochondrial membrane potential loss in BRL cells. Cells were treated with 2 $\mu \mathrm{M}$ rotenone in the presence or absence of ASD for $24 \mathrm{~h}$. Mitochondrial membrane potential was analyzed by JC-1 fluorescence. A) The fluorescence intensity ratio of JC-1 aggregates (red) to monomers (green) was calculated as an indicator of mitochondrial membrane potential. Data are expressed as means \pm S.E.M. $(\mathrm{n}=6) .{ }^{\#} P<0.01$, compared with the control group; $* P<0.05, * * P<0.01$, compared with the rotenone group. B) In live cells the dye accumulates in the mitochondria as aggregates (red), whereas in dead cells it would remain in the cytosol as monomers (green). ASD-treated BRL cells show significantly increased red fluorescence compared to the rotenoe groups. Representative photographs showing control cells (a), rotenone $(2 \mu \mathrm{M})$ (b), rotenone + ASD $(1 \mu \mathrm{M})(\mathrm{c})$, rotenone $+\operatorname{ASD}(10 \mu \mathrm{M})(\mathrm{d})$, rotenone $+\operatorname{ASD}(100 \mu \mathrm{M})(\mathrm{e})$, and ASD $(100 \mu \mathrm{M})(\mathrm{f})$.

A

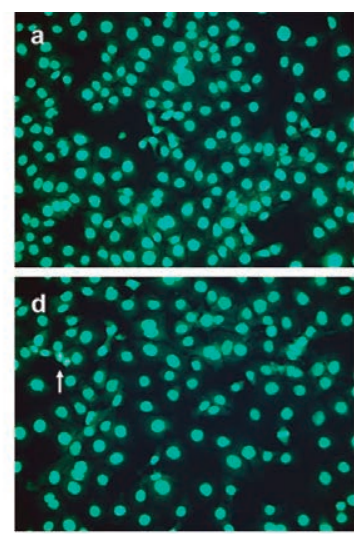

B
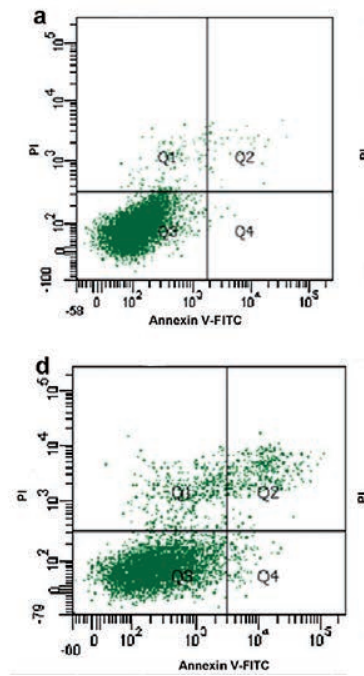
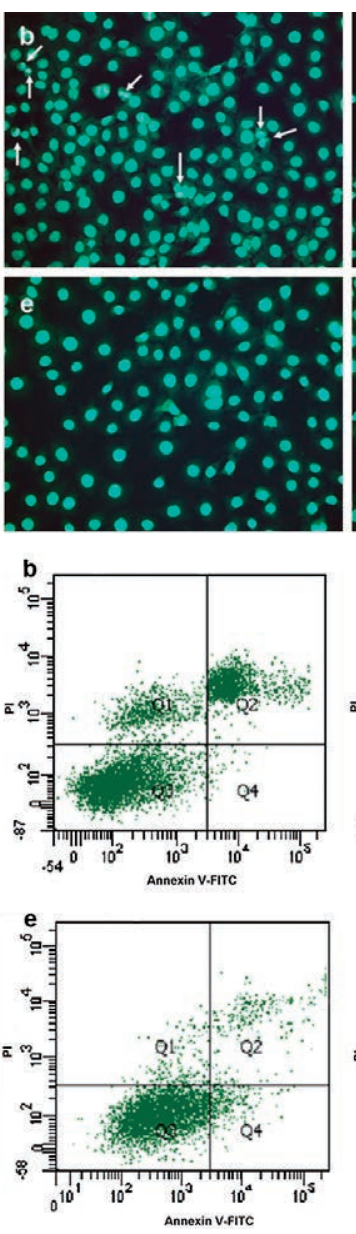
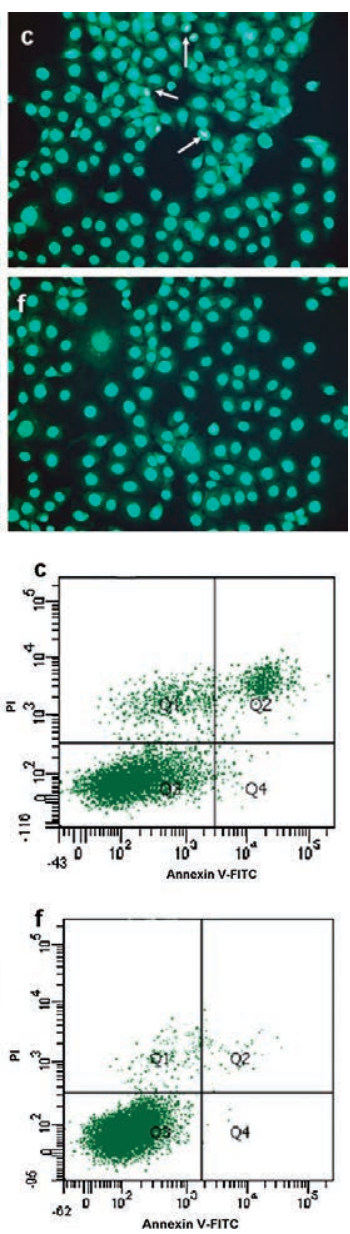

Fig. 5. Effects of Akebia saponin D (ASD) on rotenone-induced apoptosis. Cells were treated with $2 \mu \mathrm{M}$ rotenone in the presence or absence of ASD for 24 h. A) Hoechst 33342 staining was performed to visualize the apoptosis of BRL cells. Condensed or fragmented nuclei that were brightly stained were considered to be apoptotic cells. B) BRL cells were evaluated by flow cytometry after staining with Annexin V-FITC and PI. Representative photographs showing control cells (a), rotenone $(2 \mu \mathrm{M})(\mathrm{b})$, rotenone $+\operatorname{ASD}(1 \mu \mathrm{M})(\mathrm{c})$, rotenone $+\mathrm{ASD}(10 \mu \mathrm{M})(\mathrm{d})$, rotenone $+\mathrm{ASD}$ $(100 \mu \mathrm{M})(\mathrm{e})$, and ASD $(100 \mu \mathrm{M})(\mathrm{f})$. Data are expressed as means \pm S.E.M. $(n=6)$. 


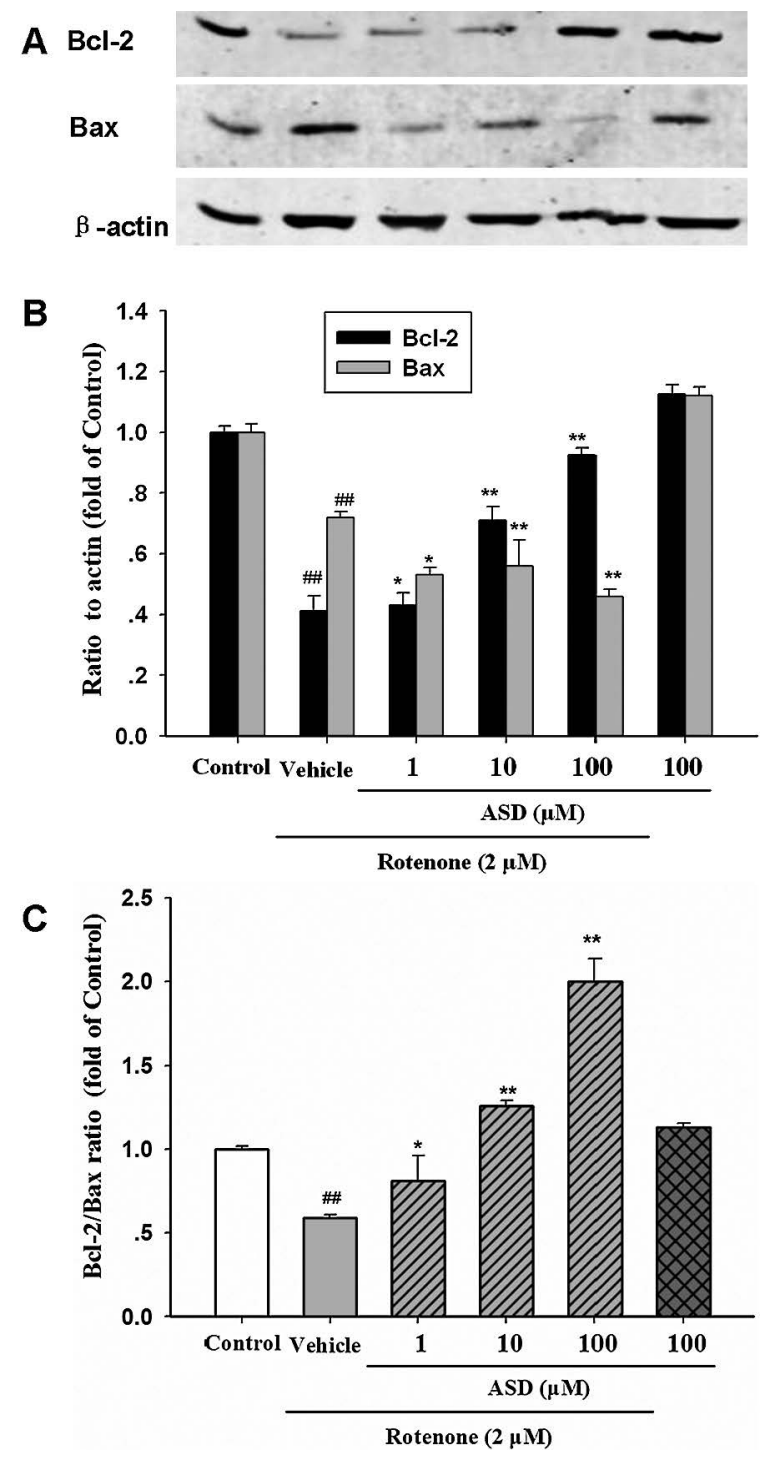

Fig. 6. Effect of Akebia saponin D (ASD) on Bcl-2 and Bax expression in BRL cells. Cells were treated with $2 \mu \mathrm{M}$ rotenone in the presence or absence of ASD for $24 \mathrm{~h}$. A) Representative western blots of $\mathrm{Bcl}-2$, Bax and $\beta$-actin. B) The quantified densitometric analysis of Bcl-2 and Bax level. C) Ratio of Bcl-2/Bax proteins. Values are means \pm S.E.M. Each experiment was conducted in triplicate, $\mathrm{n}=6$, ${ }^{\# \#} P<0.01$, compared with the control group; ${ }^{*} P<0.05$, ${ }^{*} P<0.01$, compared with the rotenone group.

\section{Discussion}

Mitochondria play a central role in regulating cell survival and death signaling in liver cells (17). Accumulating evidence indicates that mitochondrial dysfunction plays a central role in the pathogenesis of liver disorders. In the present study, the aim was to understand whether the protective mechanism of ASD involved in the mitochondrial pathway.

Rotenone, a specific inhibitor of mitochondrial com- plex I, is commonly used to investigate the drug action against liver disease. Our first task was to determine whether ASD could protect against rotenone-induced cytotoxicity in BRL liver cells and its underlying mechanisms. Exposure to $2 \mu \mathrm{M}$ rotenone for $24 \mathrm{~h}$ significantly reduced cell viability in BRL cells. The addition of ASD significantly increased cell viability in a dose-dependent manner. The direct effect of rotenone toxicity was thought to be mainly attributable to energy metabolic impairment and production of free radicals (3). Our results showed that rotenone treatment induced ATP deficiency and ROS production which were attenuated by ASD.

Mitochondria are responsible for the bulk of cellular ATP production through oxidative phosphorylation (18). Mitochondrial membrane potential $(\Delta \psi \mathrm{m})$ controls the amount of ROS produced during respiration. In this study, ASD significantly prevented rotenone induced collapse of mitochondrial membrane potential. Disruption of the mitochondrial membrane potential is one of the earliest intracellular events that occur following induction of apoptosis (19). ASD markedly prevented rotenone- induced nuclei fragmentation and decreased both apoptosis and necrosis of BRL cells. Mitochondrial integrity and the intrinsic pathway are controlled by the Bcl-2 family of proteins. Cell survival depends on the oxidation of NADH coupled to the reduction of oxygen to generate ATP by the ATP synthase. Oxygen is reduced to water by cytochromecoxidase. Rotenone inhibit the electron transport chain at cytochromecoxidase, resulting in the initiation of a death cascade through Bax (20). Bcl-2 family members are key regulators of apoptosis $(21,22)$. The interaction between the pro- and antiapoptotic Bcl-2 proteins may determine the fate of a cell, to live or to die. In this regard, the $\mathrm{Bcl}-2 / \mathrm{Bax}$ ratio may better predict the apoptotic status of the cell than the absolute concentration of either protein (23). Our result showed that treatment with ASD attenuated the rotenone-induced decrease in Bcl-2 and increase in Bax content. Thereby ASD treatment induced Bcl-2/Bax ratio increased. However, there was no significant change in the expression of Bcl-2 or Bax when ASD was treated alone. It was possible that ASD only affects damaged cells. ASD can seemingly prevent rotenoneinduced cell apoptosis through regulation of the Bcl-2/ Bax pathway.

To observe the direct effect of ASD on mitochondrial function, we used the isolated rat liver mitochondria to assess the effect of rotenone and the intervention of ASD. The primary function of mitochondria is the generation of cellular energy in the form of ATP by oxidative phosphorylation (OXPHOS). In this study we found that rotenone decreased the production of ATP. Treatment 
Table 1. Effects of Akebia saponin D (ASD) on liver mitochondrial respiratory function with L-glutamate plus L-malate or succinate as the substrate

\begin{tabular}{|c|c|c|c|c|c|}
\hline \multirow{3}{*}{ Group } & \multirow{3}{*}{$\mathrm{RCR}$} & \multicolumn{4}{|c|}{ Respiratory activity } \\
\hline & & $\mathrm{ADP} / \mathrm{O}$ & State 3 & State 4 & OPR \\
\hline & & (nM ADP / nM O) & $(\mathrm{nmol} \mathrm{O} \cdot \mathrm{m}$ & $\cdot \mathrm{mg}^{-1}$ prot$)$ & $\left(\mathrm{nM} \mathrm{ATP} \cdot \mathrm{min}^{-1} \cdot \mathrm{mg}^{-1}\right.$ prot $)$ \\
\hline \multicolumn{6}{|l|}{ With glutamate and malate } \\
\hline Control & $3.84 \pm 0.35$ & $2.64 \pm 0.11$ & $74.04 \pm 6.13$ & $19.28 \pm 1.01$ & $195.45 \pm 18.45$ \\
\hline Rotenone $(2 \mu \mathrm{M})$ & $1.32 \pm 0.15^{\# \#}$ & $1.69 \pm 0.24^{\#}$ & $28.16 \pm 11.62^{\#}$ & $21.33 \pm 1.25^{\#}$ & $47.59 \pm 13.22^{\#}$ \\
\hline ASD $(1 \mu \mathrm{M})+$ Rotenone & $1.39 \pm 0.12$ & $1.86 \pm 0.42$ & $28.42 \pm 12.08$ & $20.45 \pm 2.07$ & $52.86 \pm 11.38$ \\
\hline ASD $(10 \mu \mathrm{M})+$ Rotenone & $1.53 \pm 0.64$ & $1.99 \pm 0.21^{*}$ & $30.29 \pm 6.17$ & $19.80 \pm 2.11 *$ & $60.27 \pm 17.62 *$ \\
\hline $\operatorname{ASD}(100 \mu \mathrm{M})+$ Rotenone & $1.87 \pm 0.59 * *$ & $2.11 \pm 0.17^{*}$ & $36.72 \pm 4.74 *$ & $19.64 \pm 1.52 *$ & $77.49 \pm 21.05^{*}$ \\
\hline $\operatorname{ASD}(100 \mu \mathrm{M})$ & $3.78 \pm 0.77$ & $2.77 \pm 0.19$ & $72.68 \pm 6.35$ & $19.23 \pm 1.16$ & $201.32 \pm 25.26$ \\
\hline \multicolumn{6}{|l|}{ With succinate } \\
\hline Control & $2.69 \pm 0.13$ & $1.84 \pm 0.15$ & $94.33 \pm 5.02$ & $35.07 \pm 1.51$ & $173.56 \pm 11.38$ \\
\hline Rotenone $(2 \mu \mathrm{M})$ & $2.92 \pm 0.18$ & $1.96 \pm 0.11$ & $100.25 \pm 7.44$ & $34.33 \pm 2.03$ & $196.49 \pm 9.42$ \\
\hline ASD $(1 \mu \mathrm{M})+$ Rotenone & $2.94 \pm 0.07$ & $1.96 \pm 0.24$ & $98.76 \pm 8.08$ & $33.59 \pm 1.89$ & $193.57 \pm 12.01$ \\
\hline ASD $(10 \mu \mathrm{M})+$ Rotenone & $2.93 \pm 0.25$ & $1.99 \pm 0.41$ & $99.03 \pm 6.60$ & $33.79 \pm 3.06$ & $197.07 \pm 6.29$ \\
\hline ASD $(100 \mu \mathrm{M})+$ Rotenone & $2.95 \pm 0.33$ & $1.98 \pm 0.08$ & $99.46 \pm 4.31$ & $36.04 \pm 2.85$ & $196.93 \pm 13.21$ \\
\hline $\operatorname{ASD}(100 \mu \mathrm{M})$ & $2.88 \pm 0.34$ & $1.77 \pm 0.12$ & $95.35 \pm 7.67$ & $33.11 \pm 2.53$ & $168.77 \pm 10.06$ \\
\hline
\end{tabular}

Data for each group was expressed as mean \pm S.E.M., $\mathrm{n}=6$. Compared with control: ${ }^{\#} P<0.05,{ }^{\#} P<0.01$. Compared with rotenone: $* P<0.05$, $* * P<0.01$.
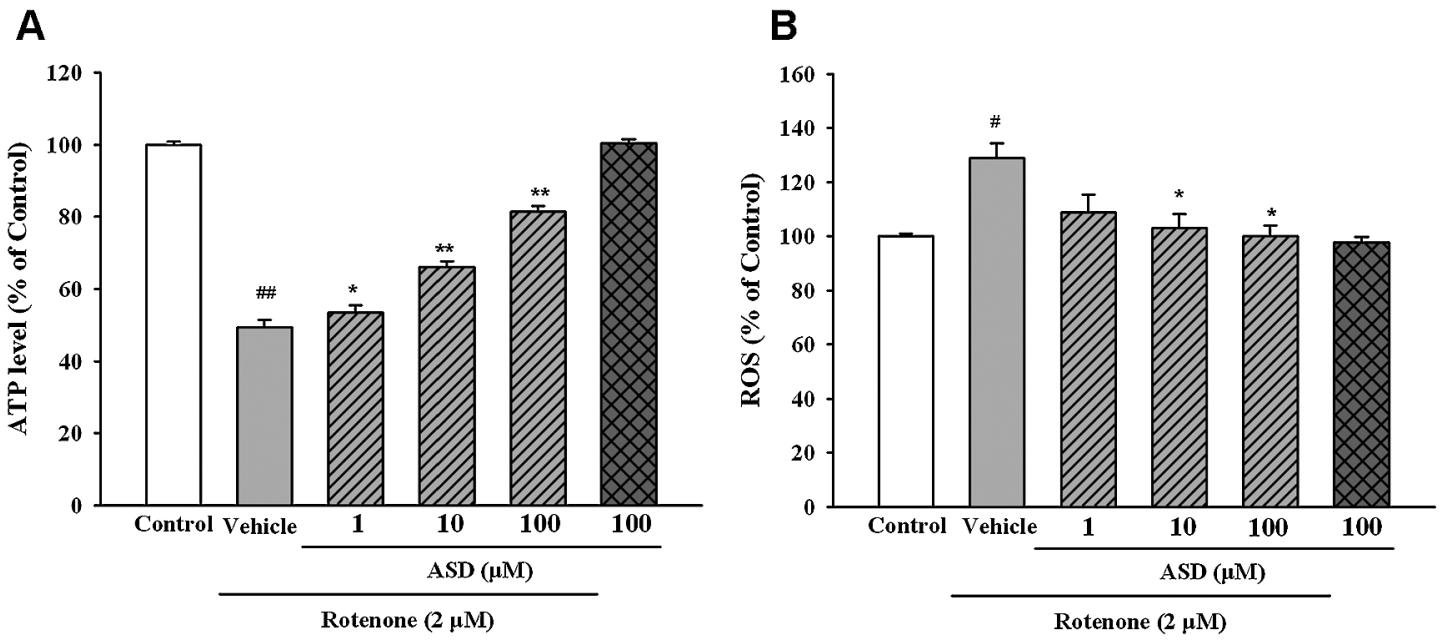

Fig. 7. Effects of Akebia saponin D (ASD) on liver mitochondrial ATP level and reactive oxidant species production. Isolated mitochondria were pretreated with vehicle or ASD for $30 \mathrm{~min}$ at $30^{\circ} \mathrm{C}$, followed by exposure to $2 \mu \mathrm{M}$ rotenone for another $30 \mathrm{~min}$. A) ATP level was assessed using a luciferase-coupled ATP quantification assay. B) Reactive oxidant species production was evaluated by DCF fluorescence. Data are expressed as means \pm S.E.M. $(\mathrm{n}=6)$. ${ }^{\#} P<0.05,{ }^{\# \#} P<0.01$, compared with the control group; $* P<0.05, * * P<0.01$, compared with the rotenone group.

with ASD attenuated rotenone-induced ATP deficiency. Mitochondria produce most of the reactive oxidant species as a byproduct of OXPHOS and are particularly vulnerable to oxidative stress. Our results showed that treatment with ASD resulted in reduction of ROS in the rotenone-damaged cells, suggesting that ASD had potent antioxidant activities. ASD significantly increased ATP and reduced ROS in both BRL cells and isolated mitochondria, which supports the notion that the target of ASD may be mitochondria.

Mitochondrial respiratory dysfunction is one of the prominent features of oxidative stress-mediated cell death. Normal mitochondrial respiration is divided into several states, with state 3 respiration coupled to ATP 
A
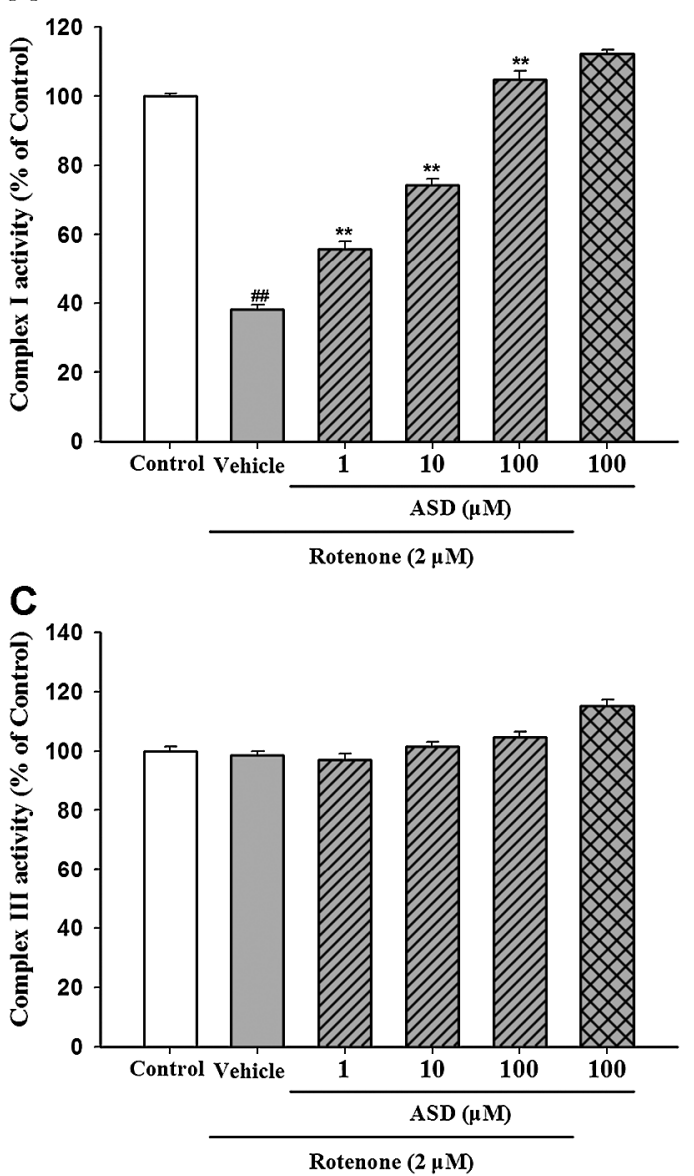

B
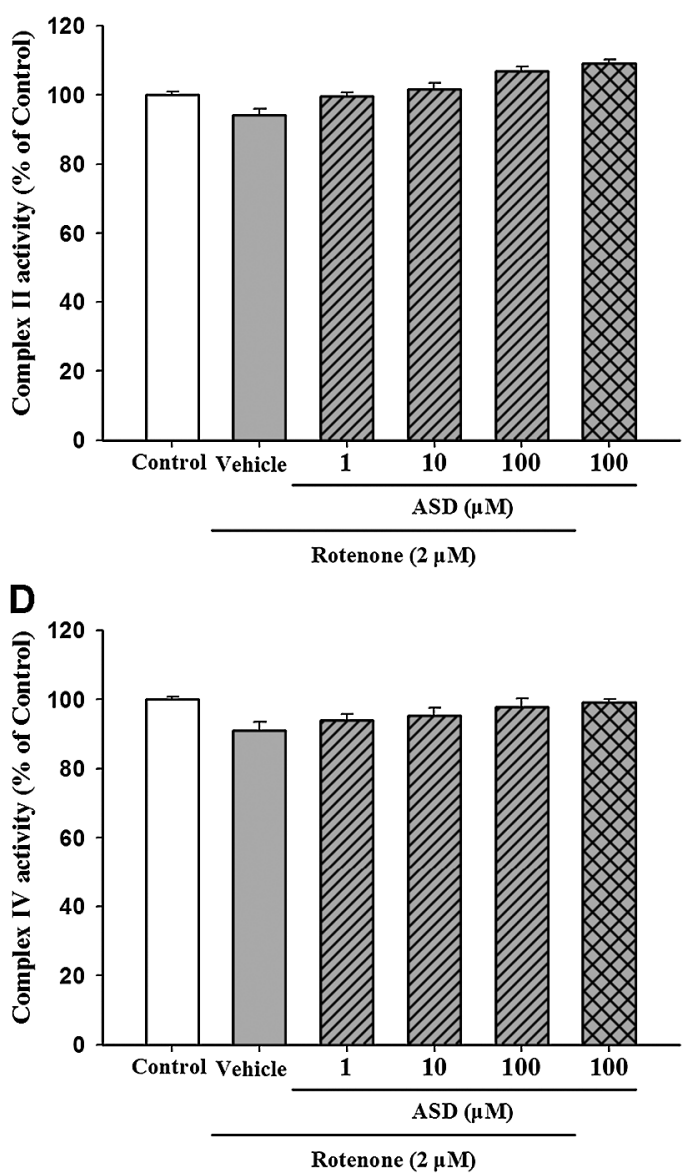

Fig. 8. Effect of Akebia saponin D (ASD) on the activity of electron transport chain complexes. The results shown are the mean \pm S.E.M. from measurements made with six preparations of mitochondria. ${ }^{\#} P<0.01$, compared with the control group; ${ }^{*} P<0.05,{ }^{* *} P<0.01$, compared with the rotenone group.

synthesis and state 4 respiration not coupled to ATP synthesis. Thus, the respiratory control ratio, which is the ratio of state 3 respiration to state 4 respiration, can be used to evaluate the functional health of mitochondria. When complex I substrates glutamate and malate were used, rotenone treatment reduced respiratory control ratio, state 3 and state 4 respiration, $\mathrm{ADP} / \mathrm{O}$, and $\mathrm{OPR}$ values. ASD raised RCR, state 3 and state 4 respiration, and $\mathrm{ADP} / \mathrm{O}$ values in the presence of glutamate and malate. When the complex II substrate succinate was used, rotenone in fact increased the oxygen consumption. When complex I activity is inhibited by rotenone, NADH oxidation and, thereby, the levels of $\mathrm{NAD}^{+}$are decreased, which in turn, impairs the oxidation of malate to oxaloacetate. Therefore, inhibitory effects of rotenone on complex I could increase complex II-linked respiration via attenuation of oxaloacetate generation. ASD significantly attenuated rotenone-induced ATP deficiency, but the degree of ASD-induced improvements for mitochon- drial parameters in the presence of glutamate and malate was very small, probably a consequence of complex I inhibition by rotenone. It is possible that the effect of ASD on mitochondrial respiration was relatively minor. ASD exerts protective actions mainly through antiapoptotic, antioxidant activity, and maintenance of cell membrane integrity.

Complex I of the electron transport chain has been reported to be the main source of superoxide production by mitochondria. To observe the direct effect of ASD on mitochondrial complex I activity, we used the isolated mitochondria to assess the effect of rotenone and the intervention of ASD. Rotenone reduced the mitochondria complex I activity but did not affect the complex II, III, or IV activity. Treatment with ASD does attenuate rotenone induced reductions in complex I activity.

In summary, ASD exerts protective action against rotenone-induced apoptosis in BRL cells and the isolated rat liver mitochondria. Regulation of the $\mathrm{Bcl}-2 / \mathrm{Bax}$ 
pathway was involved. The present study provides experimental evidence that ASD has strong antioxidant activity, and it can maintain cell membrane integrity and ameliorate mitochondrial respiratory dysfunction. It appears that ASD is a potent haptoprotectant worthy of further research and development as a candidate drug for liver disease therapy.

\section{Acknowledgments}

This study was supported by the National Natural Science Foundation of China (No. 81302822) and Basic clinical research cooperation project of Capital Medical University (No. 13JL45).

\section{Conflicts of Interest}

We declare that we have no conflict of interest.

\section{References}

1 Malhi H, Gores GJ, Lemasters JJ. Apoptosis and necrosis in the liver: a tale of two deaths? Hepatology. 2006;43(2 Suppl 1): S31-S44.

2 Li C, Gao Y, Tian J, Xing Y, Zhu H, Shen J. Long-term oral Asperosaponin VI attenuates cardiac dysfunction, myocardial fibrosis in a rat model of chronic myocardial infarction. Food Chem Toxicol. 2012;50:1432-1438.

3 Li N, Ragheb K, Lawler G, Sturgis J, Rajwa B, Melendez JA, et al. Mitochondrial complex I inhibitor rotenone induces apoptosis through enhancing mitochondrial reactive oxygen species production. J Biol Chem. 2003;278:8516-8525.

4 Teeter ME, Baginsky ML, Hatefi Y. Ectopic inhibition of the complexes of the electron transport system by rotenone, piericidin A, demerol and antimycin A. Biochim Biophys Acta. 1969;172:331-333

5 Im AR, Kim YH, Uddin MR, Chae S, Lee HW, Kim YH, et al. Betaine protects against rotenone-induced neurotoxicity in PC12 cells. Cell Mol Neurobiol. 2013;33:625-635.

6 Tamilselvam K, Braidy N, Manivasagam T, Essa MM, Prasad NR, Karthikeyan S, et al. Neuroprotective effects of hesperidin, a plant flavanone, on rotenone-induced oxidative stress and apoptosis in a cellular model for Parkinson's disease. Oxid Med Cell Longev. 2013:102741.

7 Yu X, Wang LN, Ma L, You R, Cui R, Ji D, et al. Akebia saponin $\mathrm{D}$ attenuates ibotenic acid-induced cognitive deficits and proapoptotic response in rats: involvement of MAPK signal pathway. Pharmacol Biochem Behav. 2012;101:479-486.

8 Jeong SI, Zhou B, Bae JB, Kim NS, Kim SG, Kwon J, et al. Apoptosis-inducing effect of akebia saponin D from the roots of Dipsacus asper Wall in U937 cells. Arch Pharm Res. 2008; 31:1399-1404.

9 Zhou YQ, Yang ZL, Xu L, Li P, Hu YZ. Akebia saponin D, a saponin component from Dipsacus asper Wall, protects PC 12 cells against amyloid-beta induced cytotoxicity. Cell Biol Int. 2009;33:1102-1110.

10 Li C, Liu Z, Tian J, Li G, Jiang W, Zhang G, et al. Protective roles of Asperosaponin VI, a triterpene saponin isolated from Dipsacus asper Wall on acute myocardial infarction in rats. Eur J Pharmacol. 2010;627:235-241.

11 Li C, Tian J, Li G, Jiang W, Xing Y, Hou J, et al. Asperosaponin VI protects cardiac myocytes from hypoxia-induced apoptosis via activation of the PI3K/Akt and CREB pathways. Eur J Pharmacol. 2010;649:100-107.

12 Peng LH, Ko CH, Siu SW, Koon CM, Yue GL, Cheng WH, et al. In vitro $\&$ in vivo assessment of a herbal formula used topically for bone fracture treatment. J Ethnopharmacol. 2010;131:282289.

13 Niu Y, Li Y, Huang H, Kong X, Zhang R, Liu L, et al. Asperosaponin VI, a saponin component from Dipsacus asper wall, induces osteoblast differentiation through bone morphogenetic protein-2/p38 and extracellular signal-regulated kinase 1/2 pathway. Phytother Res. 2011;25:1700-1706.

14 Li C, Zhang X-F, Lv Y-L, Lan Y-Y, Lei N, Liu L-H. Hepatoprotective effect of akebia saponin D on the acute liver injury induced by $\mathrm{CCl} 4$ in mice. West China Journal of Pharmaceutical Sciences. 2012;27:257-259.

15 Lan Y, Lei N, Zhang X, Yang R, Li C, Liu L-H. Hypolipidemic function with liver protection of extracts from Dipsacus asper on nonalcoholic fatty liver diseas e in mice and its activie fractions. Chinese Traditional and Herbal Drugs. 2011;42:2497-2501.

16 Gong LL, Fang LH, Wang SB, Sun JL, Qin HL, Li XX, et al. Coptisine exert cardioprotective effect through anti-oxidative and inhibition of RhoA/Rho kinase pathway on isoproterenolinduced myocardial infarction in rats. Atherosclerosis. 2012;222: $50-58$.

17 Singh BK, Tripathi M, Chaudhari BP, Pandey PK, Kakkar P. Natural terpenes prevent mitochondrial dysfunction, oxidative stress and release of apoptotic proteins during nimesulidehepatotoxicity in rats. PLoS One. 2012;7:e34200.

18 Galloway CA, Yoon Y. Perspectives on: SGP symposium on mitochondrial physiology and medicine: what comes first, misshape or dysfunction? The view from metabolic excess. J Gen Physiol. 2012;139:455-463.

19 Gottlieb E, Armour SM, Harris MH, Thompson CB. Mitochondrial membrane potential regulates matrix configuration and cytochrome c release during apoptosis. Cell Death Differ. 2003; 10:709-717.

20 McClintock DS, Santore MT, Lee VY, Brunelle J, Budinger GR, Zong WX, et al. Bcl-2 family members and functional electron transport chain regulate oxygen deprivation-induced cell death. Mol Cell Biol. 2002;22:94-104.

21 Brenner D, Mak TW. Mitochondrial cell death effectors. Curr Opin Cell Biol. 2009;21:871-877.

22 Youle RJ, Strasser A. The BCL-2 protein family: opposing activities that mediate cell death. Nature Rev. 2008;9:47-59.

23 Cory S, Adams JM. The Bcl2 family: regulators of the cellular life-or-death switch. Nat Rev Cancer. 2002;2:647-656. 